Proyecciones Journal of Mathematics

Vol. 36, No 3, pp. 363-372, September 2017.

Universidad Católica del Norte

Antofagasta - Chile

\title{
Edge fixed monophonic number of a graph
}

\author{
P. Titus \\ University College of Engineering, India \\ and \\ S. Eldin Vanaja \\ University College of Engineering, India \\ Received : July 2015. Accepted : May 2017
}

\begin{abstract}
For an edge $x y$ in a connected graph $G$ of order $p \geq 3$, a set $S \subseteq V(G)$ is an $x y$-monophonic set of $G$ if each vertex $v \in V(G)$ lies on an $x-u$ monophonic path or a $y-u$ monophonic path for some element $u$ in $S$. The minimum cardinality of an xy-monophonic set of $G$ is defined as the xy-monophonic number of $G$, denoted by $m_{x y}(G)$. An xy-monophonic set of cardinality $m_{x y}(G)$ is called a $m_{x y}$-set of $G$. We determine bounds for it and find the same for special classes of graphs. It is shown that for any three positive integers $r, d$ and $n \geq 2$ with $2 \leq r \leq d$, there exists a connected graph $G$ with monophonic radius $r$, monophonic diameter $d$ and $m_{x y}(G)=n$ for some edge $x y$ in $G$.
\end{abstract}

Key Words : Monophonic path, vertex monophonic number, edge fixed monophonic number.

Mathematics Subject Classification : 05C12. 


\section{Introduction}

By a graph $G=(V, E)$ we mean a finite undirected connected graph without loops or multiple edges. The order and size of $G$ are denoted by $p$ and $q$ respectively. For basic graph theoretic terminology we refer to $[1,2]$. For vertices $x$ and $y$ in a connected graph $G$, the distance $d(x, y)$ is the length of a shortest $x-y$ path in $G$. An $x-y$ path of length $d(x, y)$ is called an $x-y$ geodesic. The neighborhood of a vertex $v$ is the set $N(v)$ consisting of all vertices $u$ which are adjacent with $v$. A vertex $v$ is a simplicial vertex if the subgraph induced by its neighbors is complete. A non-separable graph is connected, non-trivial, and has no cut-vertices. A block of a graph is a maximal non-separable subgraph. A connected block graph is a connected graph in which each of its blocks is complete. A caterpillar is a tree for which the removal of all the end vertices gives a path.

A chord of a path $P$ is an edge joining two non-adjacent vertices of $P$. A path $P$ is called monophonic if it is a chordless path. The closed intervel $I_{m}[x, y]$ consists of all vertices lying on some $x-y$ monophonic of $G$. For any two vertices $u$ and $v$ in a connected graph $G$, the monophonic distance $d_{m}(u, v)$ from $u$ to $v$ is defined as the length of a longest $u-v$ monophonic path in $G$. The monophonic eccentricity $e_{m}(v)$ of a vertex $v$ in $G$ is $e_{m}(v)=\max \left\{d_{m}(v, u): u \in V(G)\right\}$. The monophonic radius, $\operatorname{rad}_{m}(G)$ of $G$ is $\operatorname{rad}_{m}\{G\}$ $=\min \left\{e_{m}(v): v \in V(G)\right\}$ and the monophonic diameter, $\operatorname{diam}_{m}\{G\}$ of $G$ is $\operatorname{diam}_{m}\{G\}$ $=\max \left\{e_{m}(v): v \in V(G)\right\}$. The monophonic distance was introduced in [3] and further studied in [4]. The concept of vertex monophonic number was introduced by Santhakumaran and Titus [5]. A set $S$ of vertices of $G$ is an x-monophonic set if each vertex $v$ of $G$ lies on an $x-y$ monophonic path in $G$ for some element $y$ in $S$. The minimum cardinality of an $x$-monophonic set of $G$ is defined as the $x$-monophonic number of $G$ and is denoted by $m_{x}(G)$ or simply $m_{x}$. An $x$-monophonic set of cardinality $m_{x}(G)$ is called a $m_{x}$-set of $G$. The following theorems will be used in the sequel.

Theorem 1.1. [2] Let $v$ be a vertex of a connected graph $G$. The following statements are equivalent:

i) $v$ is a cut-vertex of $G$.

ii) There exist vertices $u$ and $w$ distinct from $v$ such that $v$ is on every $u-w$ path.

iii) There exists a partition of the set of vertices $V-\{v\}$ into subsets $U$ and $W$ such that for any vertices $u \in U$ and $w \in W$, the vertex $v$ is on every $u-w$ path.

Theorem 1.2. [2] Every non-trivial connected graph has at least two vertices which are not cut-vertices.

Theorem 1.3. [2] Let $G$ be a connected graph with at least three vertices. The following statements are equivalent:

i) $G$ is a block.

ii) Every two vertices of $G$ lie on a common cycle.

Throughout this paper $G$ denotes a connected graph with at least three vertices. 


\section{Edge fixed monophonic number}

Definition 2.1. Let $e=x y$ be any edge of a connected graph $G$ of order at least three. A set $S$ of vertices of $G$ is an $x y$-monophonic set if every vertex of $G$ lies on either an $x-u$ monophonic path or a $y-u$ monophonic path in $G$ for some element $u$ in $S$. The minimum cardinality of an $x y$-monophonic set of $G$ is defined as the $x y$-monophonic number of $G$ and is denoted by $m_{x y}(G)$ or $m_{e}(G)$. An $x y$-monophonic set of cardinality $m_{x y}(G)$ is called a

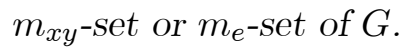

Example 2.2. For the graph $G$ given in Figure 2.1, the minimum edge fixed monophonic sets and the edge fixed monophonic numbers are given in Table 2.1.

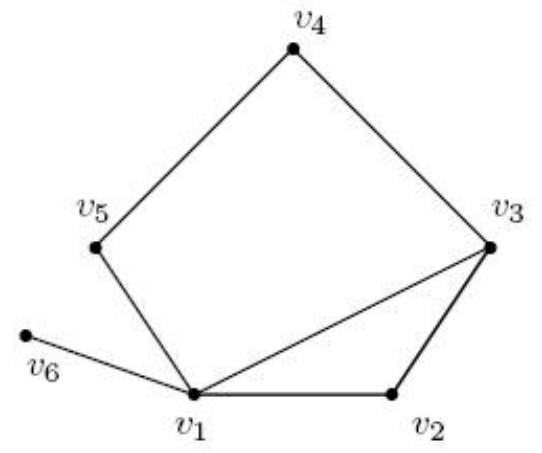

Figure 2.1: $G$

Theorem 2.3. For any edge $x y$ in a connected graph $G$ of order at least three, the vertices $x$ and $y$ do not belong to any minimum $x y$-monophonic set of $G$.

Proof. Suppose that $x$ belongs to a minimum $x y$-monophonic set, say $S$, of $G$. Since $G$ is a connected graph with at least three vertices and $x y$ in an edge, it follows from the definition of an $x y$-monophonic set that $S$ contains a vertex $v$ different from $x$ and $y$. Since the vertex $x$ lies on every $x-v$ monophonic path in $G$, it follows that $T=S-\{x\}$ is an $x y$-monophonic set of $G$, which is a contradiction to $S$ a minimum $x y$-monophonic set of $G$. Similarly, $y$ does not belong to any minimum $x y$-monophonic set of $G$. 
Table 2.1: The Edge Fixed Monophonic Number of a Graph

\begin{tabular}{|c|c|c|}
\hline $\begin{array}{c}\text { Edge } \\
e\end{array}$ & $\begin{array}{c}\text { minimum } \\
e \text {-monophonic sets }\end{array}$ & $\begin{array}{c}e \text {-monophonic } \\
\text { number }\end{array}$ \\
\hline$v_{1} v_{2}$ & $\left\{v_{4}, v_{6}\right\},\left\{v_{5}, v_{6}\right\}$ & 2 \\
\hline$v_{2} v_{3}$ & $\left\{v_{4}, v_{6}\right\},\left\{v_{5}, v_{6}\right\}$ & 2 \\
\hline$v_{3} v_{4}$ & $\left\{v_{2}, v_{6}\right\}$ & 2 \\
\hline$v_{4} v_{5}$ & $\left\{v_{2}, v_{6}\right\}$ & 2 \\
\hline$v_{5} v_{1}$ & $\left\{v_{2}, v_{6}\right\}$ & 2 \\
\hline$v_{1} v_{6}$ & $\left\{v_{2}, v_{4}\right\}$ & 2 \\
\hline$v_{1} v_{3}$ & $\left\{v_{2}, v_{6}, v_{4}\right\},\left\{v_{2}, v_{6}, v_{5}\right\}$ & 3 \\
\hline
\end{tabular}

Theorem 2.4. Let $x y$ be any edge of a connected graph $G$ of order at least three. Then

i) every simplicial vertex of $G$ other than the vertices $x$ and $y$ (whether $x$ or $y$ is simplicial or not) belongs to every $m_{x y}$-set.

ii) no cut-vertex of $G$ belongs to any $m_{x y}$-set.

Proof. (i) By Theorem 2.3, the vertices $x$ and $y$ do not belong to any $m_{x y}$-set. So, let $u \neq x, y$ be a simplicial vertex of $G$. Let $S$ be a $m_{x y}$-set of $G$ such that $u \notin S$. Then $u$ is an internal vertex of either an $x-v$ monophonic path or a $y-v$ monophonic path for some element $v$ in $S$. Without loss of generality, let $P$ be an $x-v$ monophonic path with $u$ is an internal vertex. Then both the neighbors of $u$ on $P$ are not adjacent and hence $u$ is not a simplicial vertex, which is a contradiction.

(ii) Let $v$ be a cut-vertex of $G$. Then by Theorem 1.1, there exists a partition of the set of vertices $V-\{v\}$ into subsets $U$ and $W$ such that for any vertex $u \in U$ and $w \in W$, the vertex $v$ lies on every $u-w$ path. Let $S$ be a $m_{x y}$-set of $G$. We consider three cases.

Case (i): Both $x$ and $y$ belong to $U$. Suppose that $S \cap W=\emptyset$. Let $w_{1} \in W$. Since $S$ is an $x y$-monophonic set, there exists an element $z$ in $S$ such that $w_{1}$ lies on either an $x-z$ monophonic path or a $y-z$ monophonic path in $G$. Suppose that $w_{1}$ lies on an $x-z$ monophonic path $P: x=z_{0}, z_{1}, \ldots, w_{1}, \ldots, z_{n}=z$ in $G$. Then the $x-w_{1}$ subpath of $P$ and $w_{1}-z$ subpath of $P$ both contain $v$ so that $P$ is not a path in $G$, which is a contradiction. Hence $S \cap W \neq \emptyset$. Let $w_{2} \in S \cap W$. Then $v$ is an internal vertex of any $x-w_{2}$ monophonic path and $v$ is also an internal vertex of any $y-w_{2}$ monophonic path. If $v \in S$, then let $S^{\prime}=S-\{v\}$. It is clear that every vertex that lies on an $x-v$ monophonic path also lies on an $x-w_{2}$ monophonic path. Hence it follows that $S^{\prime}$ is an $x y$-monophonic set of $G$, which is a contradiction to $S$ a minimum $x y$-monophonic set of $G$. Thus $v$ does not belong to any minimum $x y$-monophonic set of $G$.

Case (ii): Both $x$ and $y$ belong to $W$. It is simillar to Case (i).

Case (iii): Either $x=v$ or $y=v$. By Theorem 2.3, $v$ does not belong to any $m_{x y}$-set.

Corollary 2.5. Let $T$ be a tree with $k$ end vertices. Then $m_{x y}(T)=k-1$ or $k$ according as $x y$ is an end edge or cut-edge. 
Proof. This follows from Theorem 2.4.

Corollary 2.6. Let $K_{1, n}(n \geq 2)$ be a star. Then $m_{x y}\left(K_{1, n}\right)=n-1$ for any edge $x y$ in $K_{1, n}$.

Corollary 2.7. Let $G$ be a complete graph $K_{p}(p \geq 3)$. Then $m_{x y}(G)=p-2$ for any edge $x y$ in $G$.

Theorem 2.8. For any edge $x y$ in the cube $Q_{n}(n \geq 3), m_{x y}\left(Q_{n}\right)=1$.

Proof. Let $e=x y$ be an edge in $Q_{n}$ and let $x=\left(a_{1}, a_{2}, \ldots, a_{n}\right)$, where $a_{i} \in\{0,1\}$. Let $x^{\prime}=\left(a_{1}^{\prime}, a_{2}^{\prime}, \ldots, a_{n}^{\prime}\right)$ be another vertex of $Q_{n}$ such that $a_{i}^{\prime}$ is the compliment of $a_{i}$. Let $u$ be any vertex in $Q_{n}$. For convenience, let $u=\left(a_{1}, a_{2}^{\prime}, a_{3}, \ldots, a_{n}\right)$. Then $u$ lies on an $x-x^{\prime}$ monophonic path $P: x=\left(a_{1}, a_{2}, \ldots, a_{n}\right),\left(a_{1}, a_{2}^{\prime}, a_{3}, \ldots, a_{n}\right), \ldots,\left(a_{1}^{\prime}, a_{2}^{\prime}, \ldots, a_{n-1}^{\prime}, a_{n}\right),\left(a_{1}^{\prime}\right.$, $\left.a_{2}^{\prime}, \ldots, a_{n}^{\prime}\right)=x^{\prime}$. Hence $\left\{x^{\prime}\right\}$ is an $x y$-monophonic set of $Q_{n}$ and so $m_{x y}\left(Q_{n}\right)=1$.

Theorem 2.9. $\quad$ i) For any edge $x y$ in the wheel $W_{n}=K_{1}+C_{n-1}(n \geq 5)$, $m_{x y}\left(W_{n}\right)=1$.

ii) For any edge $x y$ in the complete bipartite graph $K_{m, n}(1 \leq m \leq n)$,

$$
m_{x y}\left(K_{m, n}\right)=\left\{\begin{array}{cc}
n-1 & \text { if } m=1 \\
1 & \text { if } m=2 \\
2 & \text { if } m \geq 3
\end{array}\right.
$$

Proof. (i) Let $x y$ be an edge in $W_{n}$. Then either $x$ or $y$ is a vertex of $C_{n-1}$. Let $x \in V\left(C_{n-1}\right)$ and let $z$ be a non-adjacent vertex of $x$ in $C_{n-1}$. It is clear that every vertex of $W_{n}$ lies on an $x-z$ monophonic path. Hence $\{z\}$ is a $m_{x y}$-set of $W_{n}$ and so $m_{x y}\left(W_{n}\right)=1$.

(ii) Let $U=\left\{u_{1}, u_{2}, \ldots, u_{m}\right\}$ and $W=\left\{w_{1}, w_{2}, \ldots, w_{n}\right\}$ be the vertex subsets of the bipartition of the vertices of $K_{m, n}$. If $m=1$, then by Corollary 2.6, $m_{x y}\left(K_{1, n}\right)=n-1$ for any edge $x y$ in $K_{1, n}$. If $m=2$, let $e$ be an edge in $K_{m, n}$, say $e=u_{1} w_{1}$. It is clear that every vertex of $K_{m, n}$ lies on an $u_{1}-u_{2}$ monophonic path. Hence $\left\{u_{2}\right\}$ is an $e$-monophonic set of $K_{m, n}$ and so $m_{e}\left(K_{m, n}\right)=1$. If $m \geq 3$, then it is clear that no singleton subset of $V$ is an $e$-monophonic set of $K_{m, n}$ and so $m_{e}\left(K_{m, n}\right) \geq 2$. Without loss of generality, take $e=u_{1} w_{1}$. Let $S=\left\{u_{2}, w_{2}\right\}$. Then every vertex of $U$ lies on a $w_{1}-w_{2}$ monophonic path and every vertex of $W$ lies on a $u_{1}-u_{2}$ monophonic path. Hence $S$ is an $e$-monophonic set of $K_{m, n}$ and so $m_{e}\left(K_{m, n}\right)=2$.

Theorem 2.10. For any edge $x y$ in a connected graph $G$ of order $p \geq 3$, $1 \leq m_{x y}(G) \leq p-2$.

Proof. It is clear from the definition of $m_{x y}$-set that $m_{x y}(G) \geq 1$. Also, since the vertices $x$ and $y$ do not belong to any $m_{x y}$-set, it follows that $m_{x y}(G) \leq p-2$.

Remark 2.11. The bounds for $m_{x y}(G)$ in Theorem 2.10 are sharp. If $C$ is any cycle, then $m_{x y}(C)=1$ for any edge $x y$ in $C$. For any edge $x y$ in a complete graph $K_{p}(p \geq 3)$, $m_{x y}\left(K_{p}\right)=p-2$. 
Now we proceed to characterize graphs for which the upper bound in Theorem 2.10 is attained.

Theorem 2.12. Let $G$ be a connected graph of order at least 3. Then $G$ is either $K_{p}$ or $K_{1, p-1}$ if and only if $m_{x y}(G)=p-2$ for every edge $x y$ in $G$.

Proof. If $G=K_{p}$, then by Corollary $2.7, m_{x y}(G)=p-2$ for every edge $x y$ in $G$. If $G=K_{1, p-1}$, then by Corollary $2.6, m_{x y}(G)=p-2$ for any edge $x y$ in $G$. Conversely, suppose that $m_{x y}(G)=p-2$ for every edge $x y$ in $G$. By Theorem 1.2, $G$ has at least two vertices which are not cut-vertices. Let $x y$ be an edge of $G$ with $x$ is not a cut-vertex. If $G$ has two or more cut-vertices, then by Theorem 2.4 (ii), $m_{x y}(G) \leq p-3$, which is a contradiction. Thus the number of cut-vertices $k$ of $G$ is at most one.

Case (i) $k=0$. Then the graph $G$ is a block. Now we claim that $G$ is complete. If $G$ is not complete, then there exist two vertices $x$ and $y$ in $G$ such that $d(x, y) \geq 2$. By Theorem 1.3, $x$ and $y$ lie on a common cycle and hence $x$ and $y$ lie on a smallest cycle $C: x, x_{1}, x_{2}, \ldots, y, \ldots, x_{n}, x$ of length at least 4 . Then $(V(G)-V(C)) \cup\{y\}$ is an $x x_{1^{-}}$ monophonic set of $G$ and so $m_{x x_{1}}(G) \leq p-3$, which is a contradiction. Hence $G$ is the complete graph.

Case (ii) $k=1$. Let $x$ be the cut-vertex of $G$. If $p=3$, then $G=P_{3}$, a star with three vertices. If $p \geq 4$, we claim that $G=K_{1, p-1}$. It is enough to prove that degree of every vertex other than $x$ is one. Suppose that there exists a vertex, say $y$, with $\operatorname{deg} y \geq 2$. Let $z \neq x$ be an adjacent vertex of $y$ in $G$. Let $e=y z$. Since the vertices $y$ and $z$ do not lie on any minimum $y z$-monophonic set of $G$ and by Theorem 2.4 (ii), we have $m_{y z}(G) \leq p-3$, which is a contradiction. Thus every vertex of $G$ other than $x$ is of degree one. Hence $G$ is a star.

Theorem 2.13. For any edge $x y$ in a connected graph $G$, every $x$-monophonic set of $G$ is an $x y$-monophonic set of $G$.

Proof. Let $S$ be an $x$-monophonic set of $G$. Then every vertex of $G$ lies on an $x-z$ monophonic path for some $z$ in $S$. It follows that $S$ is an $x y$-monophonic set of $G$.

Corollary 2.14. For any edge $x y$ in a connected graph $G, m_{x y}(G) \leq \min \left\{m_{x}(G), m_{y}(G)\right\}$.

Theorem 2.15. For every pair $a, b$ of integers with $1 \leq a \leq b$, there is a connected graph $G$ with $m_{x y}(G)=a$ and $m_{x}(G)=b$ for some edge $x y$ in $G$.

Proof. Let $C_{4}: x, y, z, u, x$ be a cycle of order 4 . Add $b-1$ new vertices $v_{1}, v_{2}, \ldots, v_{a-1}, w_{1}, w_{2}$, $\ldots, w_{b-a}$ and joining each $v_{i}(1 \leq i \leq a-1)$ to $x$ and joining each $w_{j}(1 \leq j \leq b-a)$ to the vertices $y$ and $u$, thereby producing the graph $G$ given in Figure 2.2. Let $S=$ $\left\{v_{1}, v_{2}, \ldots, v_{a-1}\right\}$ be the set of all simplicial vertices of $G$. Since $S$ is not an $x y$-monophonic set, it follows from Theorem 2.4(i) that $m_{x y}(G) \geq a$. On the other hand, $S_{1}=S \cup\{u\}$ 
is an $x y$-monophonic set of $G$ and so $m_{x y}(G)=\left|S_{1}\right|=a$. Clearly, $S_{2}=\left\{v_{1}, v_{2}, \ldots\right.$, $\left.v_{a-1}, z, w_{1}, w_{2}, \ldots, w_{b-a}\right\}$ is the unique $x$-monophonic set of $G$ and so $m_{x}(G)=\left|S_{2}\right|=b$.

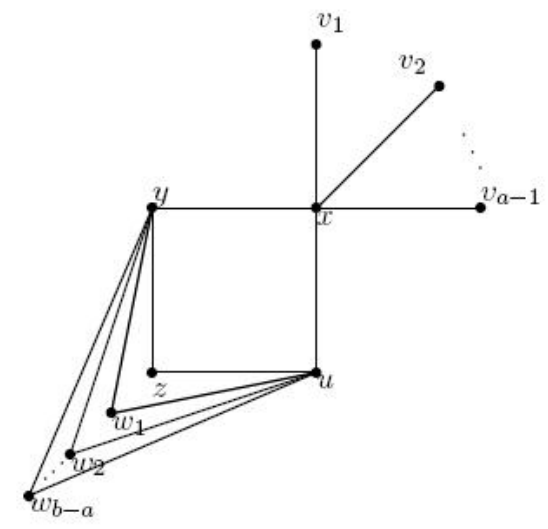

Figure 2.2: $G$

We have seen that if $G$ is a connected graph of order $p \geq 3$, then $1 \leq m_{x y}(G) \leq p-2$ for any edge $x y$ in $G$. In the following theorem we give an improved upper bound for the edge fixed monophonic number of a tree in terms of its order and monophonic diameter.

Theorem 2.16. If $T$ is a tree of order $p$ and monophonic diameter $d_{m}$, then $m_{x y}(T) \leq$ $p-d_{m}+1$ for any edge $x y$ in $T$.

Proof. Let $P: v_{0}, v_{1}, v_{2}, \ldots, v_{d_{m}}$ be a monophonic path of length $d_{m}$. Now, let $S=$ $V(G)-\left\{v_{1}, v_{2}, \ldots, v_{d_{m}-1}\right\}$. If $e$ is an internal edge of $P$, then clearly $S$ is an $e$-monophonic set of $T$ so that $m_{e}(T) \leq|S|=p-d_{m}+1$. If $e$ is an end edge of $P$, say $e=v_{0} v_{1}$, then $S_{1}=S-\left\{v_{0}\right\}$ is an $e$-monophonic set of $T$ so that $m_{e}(T) \leq\left|S_{1}\right|=p-d_{m}$. If $e=x y$ is an edge lies out side $P$, then $S_{2}=S-\{x, y\}$ is an $e$-monophonic set of $T$ so that $m_{e}(T) \leq\left|S_{2}\right|=p-d_{m}$. Hence for any edge $x y$ in $T, m_{x y}(T) \leq p-d_{m}+1$.

Remark 2.17. The bound in Theorem 2.16 is not true for any graph. For example, consider the graph $G$ given in Figure 2.3. Here $p=7, d_{m}(G)=4, m_{e}(G)=5$ and $p-d_{m}+1=4$. Hence $m_{e}(G)>p-d_{m}+1$.

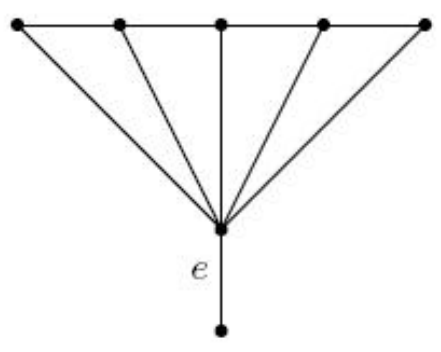

Figure 2.3: $G$

Theorem 2.18. For any edge $x y$ in a non-trivial tree $T$ of order $p$ and monophonic diameter $d_{m}, m_{x y}(T)=p-d_{m}$ or $p-d_{m}+1$ if and only if $T$ is a caterpillar. 
Proof. Let $T$ be any non-trivial tree. Let $P: v_{0}, v_{1}, \ldots, v_{d}$ be a monophonic path of length $d_{m}$. Let $k$ be the number of end vertices of $T$ and let $l$ be the number of internal vertices of $T$ other than $v_{1}, v_{2}, \ldots, v_{d-1}$. Then $d_{m}-1+l+k=p$. By Corollary 2.5, $m_{x y}(T)=k$ or $k-1$ for any edge $x y$ in $T$ and so $m_{x y}(T)=p-d_{m}-l+1$ or $p-d_{m}-l$ for any edge $x y$ in $T$. Hence $m_{x y}(T)=p-d_{m}+1$ or $p-d_{m}$ for any edge $x y$ in $T$ if and only if $l=0$, if and only if all the internal vertices of $T$ lie on the monophonic path $P$, if and only if $T$ is a caterpillar.

For any connected graph $G, \operatorname{rad}_{m}(G) \leq \operatorname{diam}_{m}(G)$. It is shown in [3] that every two positive integers $a$ and $b$ with $a \leq b$ are realizable as the monophonic radius and monophonic diameter, respectively, of some connected graph. This result can be extended so that the edge fixed monophonic number can be prescribed.

Theorem 2.19. For positive integers $r, d$ and $n \geq 2$ with $2 \leq r \leq d$, there exists a connected graph $G$ with $\operatorname{rad}_{m}(G)=r$, $\operatorname{diam}_{m}(G)=d$ and $m_{x y}(G)=n$ for some edge $x y$ in $G$.

Proof. Case (i) $2 \leq r=d$. Let $C_{r+2}: v_{1}, v_{2}, \ldots, v_{r+2}, v_{1}$ be the cycle of order $r+2$. Let $G$ be the graph obtained from $C_{r+2}$ by adding $n$ vertices $u_{1}, u_{2}, \ldots, u_{n}$ and joining each vertex $u_{i}(1 \leq i \leq n)$ to both $v_{2}$ and $v_{r+2}$, and also adding the edge $v_{1} u_{1}$. The graph $G$ is shown in Figure 2.4. It is easily verified that the monophonic eccentricity of each vertex of $G$ is $r$ and so $\operatorname{rad}_{m}(G)=\operatorname{diam}_{m}(G)=r$. Also, for the edge $v_{1} u_{1}$, it is clear that $S=\left\{v_{r+1}, u_{2}, \ldots, u_{n}\right\}$ is a minimum $x y$-monophonic set of $G$ and so $m_{x y}(G)=n$.

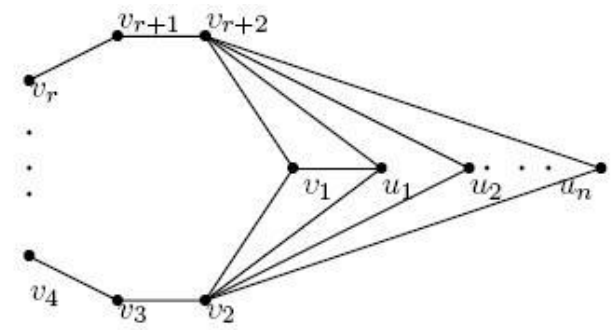

Figure 2.4:G

Case (ii) $2 \leq r<d \leq 2 r$. Let $C_{r+2}=v_{1}, v_{2}, \ldots, v_{r+2}, v_{1}$ be the cycle of order $r+2$ and let $P_{d-r+1}: u_{0}, u_{1}, \ldots, u_{d-r}$ be a path of order $d-r+1$. Let $H$ be the graph obtained from $C_{r+2}$ and $P_{d-r+1}$ by identifying $v_{1}$ in $C_{r+2}$ and $u_{0}$ in $P_{d-r+1}$. Let $G$ be the graph obtained from $H$ by adding $n-1$ new vertices $w_{1}, w_{2}, \ldots, w_{n-1}$ and joining each $w_{i}(1 \leq i \leq n-1)$ with $u_{d-r-1}$. The graph $G$ is shown in Figure 2.5. It is easily verified that $r \leq e_{m}(x) \leq d$ for any vertex $x$ in $G, e_{m}\left(v_{1}\right)=r$ and $e_{m}\left(v_{3}\right)=d$. Thus $\operatorname{rad}_{m}(G)=r$ and $\operatorname{diam}_{m}(G)=d$. For the edge $e=u_{d-r-1} u_{d-r}, S=\left\{w_{1}, w_{2}, \ldots, w_{n-1}, v_{3}\right\}$ is a minimum $e$-monophonic set of $G$ and so $m_{e}(G)=n$. 


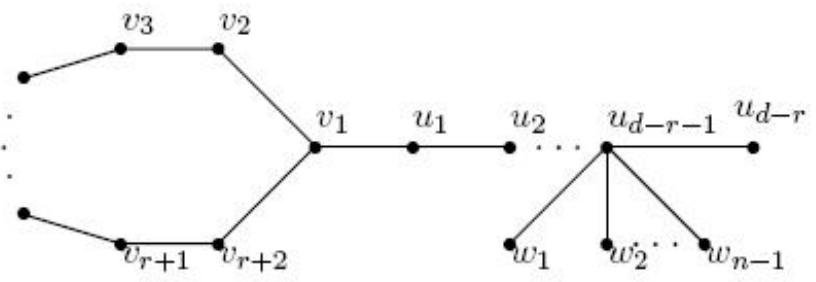

Figure 2.5: $G$

Case (iii) $d>2 r$. Let $P_{2 r-1}: v_{1}, v_{2}, \ldots, v_{2 r-1}$ be a path of order $2 r-1$. Let $G$ be the graph obtained from the wheel $W=K_{1}+C_{d+2}$ and the complete graph $K_{n}$ by identifying the vertex $v_{1}$ of $P_{2 r-1}$ with the central vertex of $W$, and identifying the vertex $v_{2 r-1}$ of $P_{2 r-1}$ with a vertex of $K_{n}$. The graph $G$ is shown in Figure 2.6. Since $d>2 r$, we have $e_{m}(x)=d$ for any vertex $x \in V\left(C_{d+2}\right)$. Also, $e_{m}(x)=2 r$ for any vertex $x \in V\left(K_{n}\right)-v_{2 r-1}$; $r \leq e_{m}(x) \leq 2 r-1$ for any vertex $x \in V\left(P_{2 r-1}\right)$; and $e_{m}(x)=r$ for the central vertex $x$ of $P_{2 r-1}$. Thus $\operatorname{rad}_{m}(G)=r$ and $\operatorname{diam}_{m}(G)=d$.

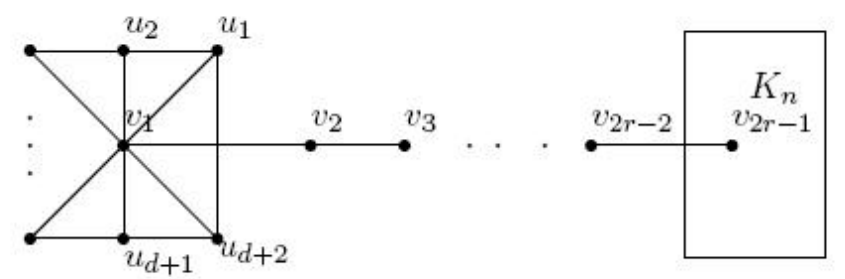

Figure 2.6:G

Let $S=V\left(K_{n}\right)-\left\{v_{2 r-1}\right\}$ be the set of all simplicial vertices of $G$. Then by Theorem 2.4(i), every $m_{e}$-set contains $S$ for the edge $e=u_{1} u_{2}$. It is clear that $S$ is not an $e$ monophonic set of $G$ and so $m_{e}(G)>|S|=n-1$. Since $S^{\prime}=S \cup\left\{u_{d+1}\right\}$ is an $e$-monophonic set of $G$, we have $m_{e}(G)=n$.

\section{References}

[1] F. Buckley and F. Harary, Distance in Graphs, Addison-Wesley, Redwood City, CA, (1990).

[2] F. Harary, Graph Theory, Addison-Wesley, Reading Mass, (1969).

[3] A. P. Santhakumaran and P. Titus, Monophonic distance in graphs, Discrete Mathematics, Algorithms and Applications, Vol. 3, No. 2, pp. 159-169, (2011).

[4] A. P. Santhakumaran and P. Titus, A note on 'Monophonic distance in graphs', Discrete Mathematics, Algorithms and Applications, Vol. 4, No. 2 (2012), DOI: 10.1142/S1793830912500188. 
[5] A. P. Santhakumaran and P. Titus, The vertex monophonic number of a graph, Discussiones Mathematicae Graph Theory, 32, pp. 189-202, (2012).

\section{P.Titus}

Department of Mathematics

University College of Engineering

Anna University Constituent College

Konam, Nagercoil - 629 004,

India

e-mail : titusvino@yahoo.com

and

\section{S. Eldin Vanaja}

Department of Mathematics

University College of Engineering

Anna University Constituent College

Konam, Nagercoil - 629 004,

India

e-mail : eldinravi28@gmail.com 